\title{
Implante de segmentos de anel estromal em ceratocone: resultados e correlações com a biomecânica corneana pré-operatória
}

\author{
Intrastromal corneal ring segments for keratoconus: \\ results and correlation with preoperative corneal biomechanics
}

Renato Ambrósio Jr',2; José Salgado Borges ${ }^{3,4}$; Cláudia Costa-Ferreira ${ }^{3,4}$; Vinícius Coelho²; Renata Siqueira da Silva ${ }^{1,2}$; Bruno Valbon ${ }^{1,2}$; Jorge Augusto Siqueira ${ }^{2}$; Guillermo Coca Velarde ${ }^{5}$

\section{Resumo}

Objetivo: Verificar os resultados da cirurgia de implante de segmentos de anel estromal para ceratocone e as possíveis correlações existentes entre o resultado cirúrgico e o estado biomecânico da córnea no pré-operatório. Métodos: Dezenove olhos de 19 pacientes com ceratocone foram avaliados antes e 12 meses após a cirurgia de implante de segmento anel estromal. O implante de um ou dois segmentos de anel de Ferrara foi realizado de acordo com o nomograma, considerando-se espessura da córnea, padrão topográfico da ectasia e o astigmatismo. O teste de Wilcoxon signed rank foi realizado para verificar diferenças entre a acuidade visual (logMAR) corrigida com óculos antes da cirurgia (AVccPré) e a obtida sem correção (AVscPós) e com correção (AVccPós) após a cirurgia, bem como entre os valores pré e pós-operatórios de variáveis tomográficas medidas com o Pentacam e variáveis biomecânicas da córnea medidas com o ORA (ocular response analyzer). As diferenças absolutas antes e após a cirurgia (valores delta $-\Delta$ ) das variáveis com diferenças significantes foram calculadas. Os valores $\Delta$ foram correlacionados com as medidas do ORA no pré-operatório por meio dos testes de Pearson ou de Spaerman, de acordo com a distribuição das variáveis ser normal ou não (teste de Kolmogorov-Smirnov). Resultados: Observou-se melhora significante na AVcc, bem como na AVscPós em comparação com a AVccPré (teste de Wilcoxon, $\mathrm{p}<0,05)$. Houve redução significativa do astigmatismo ceratométrico (AST), dos valores ceratométricos centrais (K1 e K2), do valor ceratométrico mais elevado (KMáx) e dos índices CKI (índice de severidade do ceratocone - center keratoconus index ), IHD (índice de descentração da elevação - index of height decentration) e ISV (índice de variação da superfície - index of surface variance) após a cirurgia (teste de Wilcoxon, $\mathrm{p}<0,05$ ). Observou-se um significativo aumento da espessura corneana a 3,8 e a $4 \mathrm{~mm}$ do ápice em direção temporal no meridiano horizontal, bem como houve deslocamento inferior significativo da posição do KMáx (teste de Wilcoxon, $\mathrm{p}<0,05$ ). Nenhuma variável biomecânica apresentou diferença estatisticamente significante após a cirurgia (teste de Wilcoxon, $\mathrm{p}>0,05$ ). Os parâmetros do ORA derivados das pressões de aplanamento: histerese da córnea (corneal hysteresis - CH), fator de resistência da córnea (corneal resistance factor - CRF) e as medidas da pressão ocular (IOPg e IOPcc) não apresentaram correlações significantes com os parâmetros delta $(\Delta)$. Quinze das 38 variáveis biomecânicas derivadas do sinal de reflexo da córnea do ORA apresentaram correlação significante com pelo menos um parâmetro $\Delta$, destacando-se as correlações entre o $\Delta$ KMáx com aindex e path1 (teste de Pearson, $\mathrm{p}<0,05 ; \mathrm{r}=-0,56$ e 0,56 respectivamente) e entre o $\Delta$ AVccPré-AVscPós e aindex (teste de Pearson, $\mathrm{p}<0,05 ; \mathrm{r}=0,48$ ). A variável que teve maior número de correlações significantes foi w21, que se correlacionou negativamente com $\Delta K 2, \Delta K 1, \Delta C K I$ e $\Delta K M a ́ x$ (teste de Spearman, $p<0,05 ; \mathrm{r}=-0,56 ;-0,55$; -0,51 e -0,47) respectivamente. Conclusão: Existe melhora significante da acuidade visual e de parâmetros ceratométricos após cirurgia de implante de segmentos de anel de Ferrara para ceratocone. A melhora de diversos destes parâmetros com a cirurgia foi significativamente correlacionada com características biomecânicas derivadas do sinal do reflexo da córnea do ORA no préoperatório. Um estado biomecânico pré-operatório "mais fraco" ou "menos resistente" foi significativamente relacionado com um maior benefício clínico da cirurgia. Análises com associações múltiplas (multivariadas) são necessárias, incluindo parâmetros tomográficos da córnea e de aberrometria total do pré-operatório. Tais informações abrem novos horizontes para a seleção de pacientes e o planejamento de cirurgia de implante de segmentos de anel para ceratocone.

Descritores: Ceratocone/cirurgia; Córnea/fisiologia; Próteses e implantes; Acuidade visual; Período pré-operatório; Resultado de tratamento

\footnotetext{
${ }^{1}$ Instituto de Olhos Renato Ambrósio - Rio de Janeiro (RJ), Brasil;

${ }^{2}$ Grupo de Estudos de Tomografia e Biomecânica de Córnea do Rio de Janeiro - Rio de Janeiro (RJ), Brasil;

${ }^{3}$ Serviço de Oftalmologia do Hospital de São Sebastião, Santa Maria da Feira, Portugal;

${ }^{4}$ Universidade Fernando Pessoa, Porto, Portugal.

${ }^{5}$ Departamento de Estatística, Universidade Federal Fluminense - Rio de Janeiro (RJ), Brasil.

Trabalho ganhador do 39 Prêmio Varilux - SBO 2011 na Categoria Master
}

Os autores declaram inexistir conflitos de interesse

Recebido para publicação em 4/4/2011 - Aceito para publicação em 30/6/2011 


\begin{abstract}
Objective: To verify possible correlations between the biomechanical state of the cornea before surgery and the results of stromal ring segments implant for keratoconus. Methods: Nineteen eyes of 19 patients with keratoconus were evaluated before and 12 months after stromal ring segment implant surgery. The implant of one or two Ferrara ring segments was performed accordingly to the nomogram, considering corneal thickness, ectasy pattern on topography and astigmatism. The Wilcoxon test was performed to verify the differences between visual acuity (logMAR) with correction before surgery (AVccPre) and without correction (AVscPos) and with correction (AVccPos) after surgery, topography variables measured with Pentacam on pre and post operative and corneal biomechanics variables measure with ORA (ocular response analyzer). The absolute differences before and after the surgery (delta values $-\Delta$ ) of variables with significant differences were calculated. The $\Delta$ values were correlated with the ORA measurements pre-operatively using Pearson or Spearman's tests, according with the distribution of the variables to be or not normal (Kolmogorov-Smirnov test). Results: There were significant improvements on AVcc and AVscPos comparing with AVccPre (Wilcoxon test, $\mathrm{p}<0,05$ ). Significant reduction was observed on keratometric astigmatism (AST), Keratometric central values (K1 and K2), higher keratometric value (KMax) and topometric indices: CKI (Center Keratoconus Index), IHD (Index of Height Decentration) and ISV (Index of Surface Variance) after surgery (Wilcoxon test, $\mathrm{p}<0.05)$. There was a significant increase in corneal thickness of 3.8 and $4 \mathrm{~mm}$ from the apex toward the temporal horizontal meridian observed a significant increase on cornea thickness, and there was significant downward displacement of the position of KMax (Wilcoxon test, $\mathrm{p}<0.05$ ). No variable biomechanical statistically significant difference after surgery (Wilcoxon test, $\mathrm{p}>0.05)$. The ORA parameters derivate form the aplanation pressure: Corneal Hysteresis $(\mathrm{CH})$, Corneal Resistance Factor (CRF) and the measures of the ocular pressure (IOPg and IOPcc) showed no significant correlations with the parameters delta $(\Delta)$. Fifteen of the 38 biomechanical variables derived from the sign of ORA corneal reflex presented significant correlation with at least one parameter $\Delta$, highlighting the correlations between $\Delta \mathrm{KMax}$ with aindex e path1 (Pearson test, $\mathrm{p}<0.05 ; \mathrm{r}=-0.56$ and 0.56 respectively) and between AVccPre-AVscPos and aindex (Pearson test, $\mathrm{p}<0.05 ; \mathrm{r}=0.48$ ). The variable that had the greatest number of significant correlations was w21, which correlated negatively with, $\Delta \mathrm{K} 2, \Delta \mathrm{K} 1, \Delta \mathrm{CKI}$ and $\Delta \mathrm{KMax}$ (Spearman test, $\mathrm{p}<0.05 ; \mathrm{r}=-0.56 ;-0.55 ;-0.51$ and -0.47$)$ respectively. Conclusion: There is a significant improvement in visual acuity and keratometric parameters after surgery of implant the Ferrara ring segments for keratoconus. The improvement of several of these parameters with the surgery was significantly correlated with biomechanical characteristics derived from the signal of the ORA corneal reflex preoperatively. A biomechanical preoperative state "weaker" or "less resistant" was significantly related to greater clinical benefit of surgery. Analysis with multiple associations (multivariate) is necessary, including topographic parameters of the cornea and wavefront analysis of preoperative total. This information opens up new horizons for patient selection and planning of ring segments implant surgery for keratoconus.
\end{abstract}

Keywords: Keratoconus/surgery; Cornea/physiology; Prostheses and implants; Visual acuity; Preoperative period; Treatment outcome

\section{INTRODUÇÃO}

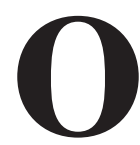
implante de segmento de anel estromal é uma alternativa cirúrgica para tratamento de ceratocone. ${ }^{(1-20)}$ Tradicionalmente, a cirurgia é indicada para adiar ou mesmo evitar o transplante de córnea em casos em que a reabilitação visual não é possível com métodos tradicionais (óculos e lentes de contato $[\mathrm{LC}])^{(2,7,12,13,19,20)}$ Entretanto, existe uma tendência para indicações mais precoces e de forma eletiva (ex. casos ainda com boa acuidade visual com LC), ${ }^{(13-18)}$ havendo uma exigência crescente para maior previsibilidade e segurança para estes procedimentos.

Embora os resultados com estes procedimentos sejam altamente satisfatórios, existe uma grande variabilidade nos resultados obtidos. Por exemplo, é comum observarmos que dois casos com ceratocone apresentado características topográficas similares tenham resultados distintos apesar de ter um mesmo plano de cirurgia realizada por cirurgião experiente. Os nomogramas classicamente levam em consideração o aspecto topográfico (curvatura axial) da ectasia, a espessura da córnea e o estado refracional do paciente. ${ }^{(7,13,19)}$

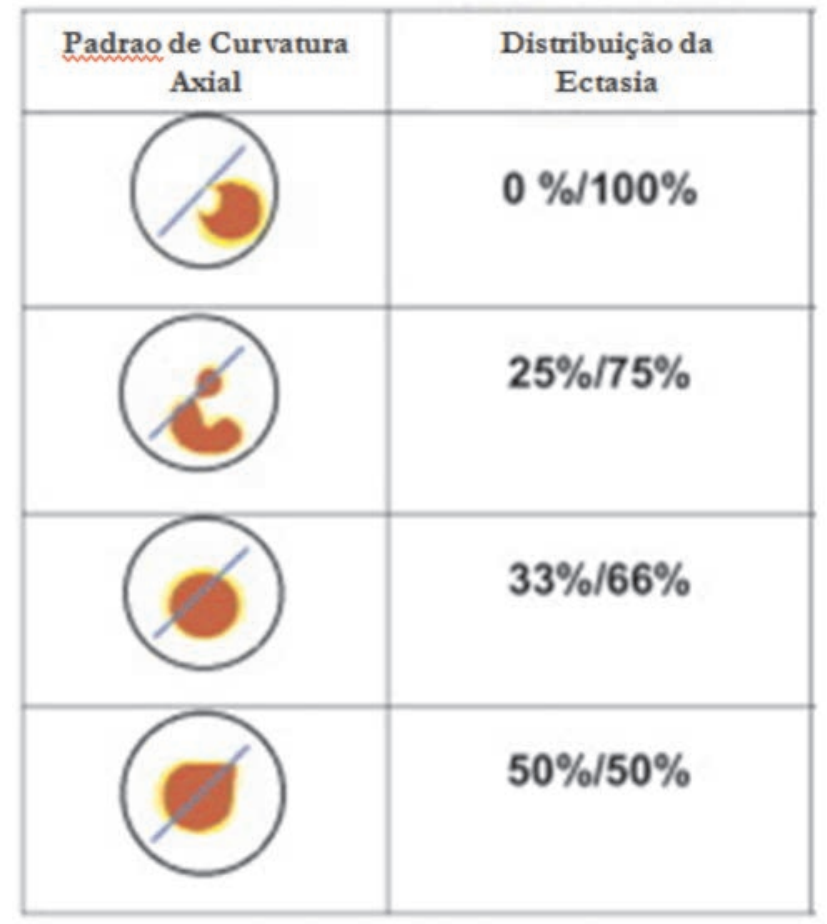

Figura 1: Padrão de distribuição da ectasia no mapa de curvatura (axial ou sagital) da topografia 
Nomogramas mais avançados são propostos com base em novas variáveis, como cálculos da aberrometria (decomposição dos polinômios de Zernike) da córnea ${ }^{21} \mathrm{e}$ estudos vetoriais do astigmatismo. ${ }^{(22)}$ Outras variáveis como a asfericidade da córnea vêm sendo estudadas para aprimorar os nomogramas de implante de anel, sendo esta uma das áreas de maior interesse da subespecialidade.

Desde o advento da topografia corneana, ${ }^{(23,24)}$ que estuda detalhadamente a face anterior da córnea, novas tecnologias incrementam a propedêutica para caracterização da córnea em nível tomográfico, ${ }^{(25,26)}$ biomecânico, ${ }^{(27-29)}$ bem como das aberrações de alta ordem do sistema óptico ocular. ${ }^{(24,30)}$ Variáveis geradas por estes exames mais avançados permitem, além de aumentar nossa capacidade de diagnóstico e prognóstico de ceratocone e outras ectasias, melhorar o planejamento cirúrgico e o seguimento dos resultados obtidos.

Considerando-se a variabilidade dos resultados obtidos com implante de segmento de anel para ceratocone, levantamos a hipótese que o comportamento pós cirúrgico está relacionado com o estado biomecânico da córnea. O presente estudo reporta os resultados com um ano de seguimento de uma série de casos de ceratocone tratados cirurgicamente com implante de um ou dois segmentos de anel de Ferrara. Os resultados foram correlacionados com características biomecânicas pré-operatórias da córnea obtidas com o ORA (ocular response analyzer, Reichert).

\section{MÉTODOS}

O estudo retrospectivo incluiu 19 olhos de 19 pacientes com ceratocone, tratados com implante de um ou dois segmentos de anel de Ferrara. Os pacientes foram operados pelo mesmo cirurgião (JSB). O estudo seguiu os critérios e recomendações da Declaração de Helsinki. Os pacientes apresentavam ceratocone com grau II ou III (Amsler-Krumeich), eram intolerantes ao uso de lentes de contato e concordaram com os riscos, benefícios e limitações da cirurgia proposta. Todos os pacientes assinaram termo de consentimento e concordaram em participar deste estudo. Os critérios de exclusão para a cirurgia foram opacidade central da córnea e espessura inferior a $370 \mu \mathrm{m}$ ou a $460 \mu \mathrm{m}$ no local da incisão.

O implante de um ou dois segmentos de $160^{\circ}$ de arco anel de Ferrara, que apresenta formato triangular com $600 \mu \mathrm{m}$ de base, raio interno $5 \mathrm{~mm}$ e raio externo $6 \mathrm{~mm}$, foi realizado de acordo com nomograma em vigência. $\mathrm{O}$ nomograma utilizado considerou a espessura da córnea e o padrão topográfico da ectasia no mapa de curvatura axial ou sagital e o astigmatismo. $\mathrm{O}$ implante de um ou dois segmentos e a distribuição das espessuras destes foi definida com base na distribuição ceratométrica da ectasia, dividindo-se a córnea em metades (Figura 1). Por exemplo, se $100 \%$ da área de maior curvatura no mapa de curvatura axial estivesse localizado em uma metade, apenas um seguimento seria implantado. Se $25 \%$ ou mais estivesse na outra metade, um segundo seguimento era utilizado, mantendo-se a relação das espessuras dos seguimentos o mais semelhante possível com a da distribuição da área de maior curvatura.

As cirurgias foram realizadas pelo mesmo cirurgião sob anestesia tópica. A profundidade da incisão foi de $80 \%$ da espessura no local da incisão de acordo com o mapa paquimétrico (Pentacam). Uma incisão única superior foi realizada. A marcação do trajeto do anel com tinta de violeta de Genciana foi centralizada no primeiro reflexo de Purkinje da córnea com fixação do paciente no feixe de luz do microscópio. Após incisão com bisturi de diamante, a dissecção foi realizada de forma manual com criação de um bolso inicial com dissector de Soares, seguindo-se com confecção do túnel com espátulas curvas de Ferrara para única incisão. O implante de um ou dois segmentos de anel foi realizado com pinça e gancho tipo Sinskey. Associação de colírio antibiótico e corticóide foi usada por uma semana. Lubrificantes e tratamento antialérgico tópicos foram prescritos de acordo com cada caso.

Os pacientes foram avaliados antes e após 12 meses da cirurgia. Os pacientes foram submetidos, antes e após a cirurgia, a exame oftalmológico geral, incluindo medidas da acuidade visual sem e com correção, biomicroscopia, fundoscopia, refratometria, tonometria e estudo biomecânico da córnea com o ORA (Reichert) e tomografia de córnea e segmento anterior com o Pentacam (Oculus). A acuidade visual (AV) foi convertida de frações de Snellen com numerador 20 para $\log$ MAR. A AV corrigida com melhor correção antes da cirurgia (AVccPré) foi comparada com a obtida sem correção (AVscPós) e com correção (AVccPós) após a cirurgia. Cinco índices topométricos, calculados a partir de dados do mapa de curvatura axial anterior (Tabela 1) foram estudados juntamente com a ceratometria central (K1 e K2), o valor de ceratometria mais elevado (KMáx) e sua posição cartesiana (x [sempre positivo na direção nasal] e y [positivo superior]) em relação ao ponto central dos mapas (ápice).

Além das variáveis topométricas, a espessura central, a do ponto mais fino e as espessuras ao longo do meridiano horizontal passando pelo ponto mais fino com 


\section{Tabela 1}

\section{Variáveis topométricas do Pentacam}

\begin{tabular}{lll}
\hline CKI & Center Keratoconus Index & $\begin{array}{l}\text { índice que combina valores ceratométricos para } \\
\text { diagnóstico de ceratocone }\end{array}$ \\
ISV & $\begin{array}{l}\text { Index of Surface Variation } \\
\text { variação da curvatura em relação ao valor médio } \\
\text { simetria superior e inferior dos valores } \\
\text { ceratométricos }\end{array}$ \\
IHA & $\begin{array}{l}\text { Index of Height Asymmetry } \\
\text { simetria superior e inferior dos valores de } \\
\text { elevação } \\
\text { valor de descentralizacao dos dados de elevação } \\
\text { no eito vertical }\end{array}$
\end{tabular}

$0,2 \mathrm{~mm}$ de intervalo foram estudadas, juntamente com os índices de progressão paquimétrica.

Além dos parâmetros básicos do ORA derivados das pressões de aplanamento: histerese da córnea (Corneal Hysteresis - CH), fator de resistência da córnea (Corneal Resistance Factor - CRF) e as medidas da pressão ocular (IOPg e IOPcc), ${ }^{(29)} 38$ variáveis biomecânicas derivadas do sinal de reflexo da córnea do ORA foram estudadas. Estas 38 novas variáveis foram introduzidas por David Luce, $\mathrm{PhD}$ com o objetivo de descrever numericamente o comportamento da deformação da córnea em resposta ao sopro de ar durante o exame do ORA (Tabela 2, Figura 2).

$\mathrm{O}$ teste de Wilcoxon signed rank para grupos pareados com distribuição não paramétrica foi realizado para verificar diferenças de cada parâmetro estudado antes e após a cirurgia. As diferenças absolutas (valores delta $-\Delta$ ) entre as variáveis com diferenças significantes antes e após a cirurgia foram calculadas. Quanto maior o valor $\Delta$, maior o benefício com a cirurgia, com exceção dos valores de espessura. Por exemplo, a melhora da AV em logMAR apresenta redução dos valores, assim como o deslocamento vertical do KMáx, que se apresenta mais negativo (mais deslocado inferiormente) após a cirurgia (Figura 3). As reduções do astigmatismo e dos valores ceratométricos são associadas com melhores resultados, também determinando valores $\Delta$ positivos. Os valores $\Delta$ foram correlacionados de forma pareada para cada caso com as medidas do ORA no pré-operatório por meio dos testes de Pearson ou de Spearman, de acordo com a distribuição das variáveis ser normal ou não (teste de Kolmogorov-Smirnov). Caso ambas as variáveis, valor $\Delta$ e o parâmetro do ORA pré-operatório, tivessem distribuição normal, o teste de Pearson era utilizado. No caso de uma das variáveis correlacionadas não apresentar distribuição normal, o teste de Spearman era utilizado. Valores de p menores que 0,05 foram considerados como estatisticamente significantes.
Tabela 2

Variáveis do ORA

\begin{tabular}{cc} 
IOPg & path1 \\
IOPcc & path2 \\
CRF & mslew1 \\
CH & mslew2 \\
LTI & slew1 \\
aindex & slew2 \\
bindex & aplhf \\
p1area & p1area1 \\
p2area & p2area1 \\
aspect1 & aspect11 \\
aspect2 & aspect21 \\
uslope1 & uslope11 \\
uslope2 & uslope21 \\
dslope1 & dslope11 \\
dslope2 & dslope21 \\
w1 & w11 \\
w2 & w21 \\
h1 & h11 \\
h2 & h21 \\
dive1 & path11 \\
\hline
\end{tabular}

\section{Resultados}

Quatorze pacientes eram do sexo masculino e 5 pacientes do sexo feminino. A idade variou entre $24 \mathrm{e}$ 46 anos $(31.9 \pm 6.2)$. De acordo com o nomograma, considerando-se a distribuição da ectasia no mapa de curvatura axial anterior, optou-se pela colocação de um segmento de anel em 10 olhos e dois segmentos em 9 casos. O acompanhamento pós-operatório médio foi de $18 \pm 4,3$ meses, variando entre 16 e 27 meses.

Observou-se melhora significante na AVcc antes e após a cirurgia, bem como na AVscPós em comparação com a AVccPré (teste de Wilcoxon, $p<0,001$ e p=0,03). A média da acuidade visual corrigida (AVcc) melhorou de $0,4 \pm 0,28(20 / 50)$ para $0,11 \pm 0,11$ (20/26). A média da 
Tabela 3

Variáveis topométricas com diferenças significante antes e 12 meses após cirurgia de implante de segmentos de anel de Ferrara (*Teste de Wilcoxon)

\begin{tabular}{lccccccc}
\hline & K1 (D) & K2 (D) & ASTIG & K Max & CKI & IHD & ISV \\
\hline valor p $^{*}$ & 0,025 & 0,008 & 0,028 & 0,027 & 0,007 & 0,032 & 0,014 \\
Média Pré & 48,484 & 53,826 & 5,54 & 60,375 & 1,093 & 0,121 & 122,368 \\
Desv. Pad. Pré & 3,723 & 4,907 & 3,02 & 6,081 & 0,041 & 0,044 & 32,733 \\
Média Pós & 45,911 & 49,495 & 3,44 & 55,613 & 1,054 & 0,090 & 93,47 \\
Desv. Pad. Pós & 3,017 & 4,615 & 2,61 & 6,635 & 0,043 & 0,040 & 34,835 \\
\hline
\end{tabular}

Tabela 4

Sumário de todas as correlações significantes entre os valores $\Delta$ e os parâmetros biomecânicos pré-operatórios

\begin{tabular}{ccccc}
\hline $\begin{array}{c}\text { Melhora com cirurgia } \\
\text { (Valor } \Delta \text { ) }\end{array}$ & $\begin{array}{c}\text { Parâmetro } \\
\text { biomecânico pré-op }\end{array}$ & Teste & Valor p & $\begin{array}{c}\text { Coeficiente } \\
\text { de correlação }\end{array}$ \\
\hline KMáx & aindex & Pearson & 0,0115 & -0.5664 \\
AVccPréx AVscPós & aindex & Pearson & 0,036 & 0,4835038 \\
KMáx_y & aspect11 & Pearson & 0,0226 & 0,5195514 \\
KMáx_y & aspect21 & Pearson & 0,0399 & 0,4749466 \\
ASTIG & bindex & Spearman & 0,0364 & $-0,4922805$ \\
ASTIG & LTI & Pearson & 0,0428 & $-0,4690226$ \\
KMáx_y & mslew1 & Pearson & 0,0317 & 0,4936497 \\
KMáx & plareal & Pearson & 0,0407 & $-0,4733162$ \\
Paqui temp. a 4mm ápice & p2area & Spearman & 0,0412 & 0,4820019 \\
Paqui temp. a 3,8mm ápice & p2area & Spearman & 0,044 & 0,4756473 \\
KMáx & path1 & Pearson & 0,0119 & 0,5637977 \\
K2.D & Slew1 & Pearson & 0,0346 & 0,4866947 \\
KMáx_y & slew1 & Pearson & 0,0361 & 0,4831907 \\
ASTIG & slew2 & Pearson & 0,0489 & $-0,457487$ \\
KMáx_y & $w 1$ & Pearson & 0,0414 & $-0,4718817$ \\
KMáx_y & $w 11$ & Pearson & 0,0175 & $-0,5379393$ \\
K1 & $w 2$ & Pearson & 0,041 & $-0,4726129$ \\
CKI & $w 2$ & Pearson & 0,048 & $-0,4590463$ \\
K2 & $w 21$ & Spearman & 0,0171 & $-0,5610617$ \\
K1 & $w 21$ & Spearman & 0,0198 & $-0,5483064$ \\
CKI & $w 21$ & Spearman & 0,0296 & $-0,5117734$ \\
KMáx & $w 21$ & Spearman & 0,0459 & $-0,4696427$ \\
\hline
\end{tabular}

acuidade visual sem correção (AVscPós) pós foi de $0,29 \pm 0,26$ (20/39). Houve também diferença significante entre a AVccPós e AVscPós (teste de Wilcoxon, $\mathrm{p}=0,009$ ).

Houve redução significativa do astigmatismo ceratométrico (AST), dos valores ceratométricos centrais (K1 e K2), do astigmatismo (ASTIG), do valor ceratométrico mais elevado (KMáx) e dos índices CKI (Center Keratoconus Index), IHD (Index of Height Decentration) e ISV (Index of Surface Variance) após a cirurgia (Tabela 3).
Observou-se um significativo aumento da espessura corneana a 3,8 e a 4,0mm do ápice em direção temporal no meridiano horizontal (teste de Wilcoxon, $\mathrm{p}<0,05)$. A espessura média da córnea aumentou de $603 \pm 36$ para $627 \pm 36$ a $3,8 \mathrm{~mm}$ e de $615 \pm 36$ para $640 \pm 37$ a $4 \mathrm{~mm}$.

Observou-se deslocamento inferior significativo da posição do KMáx (teste de Wilcoxon, $\mathrm{p}<0,05$ ). A posição do KMáx no eixo vertical (Y) passou de $-1,09 \pm 0,84$ para $-1,85 \pm 1,22$ (Figura 3). 


\section{Figura 2 - Análise do Sinal do ORA}
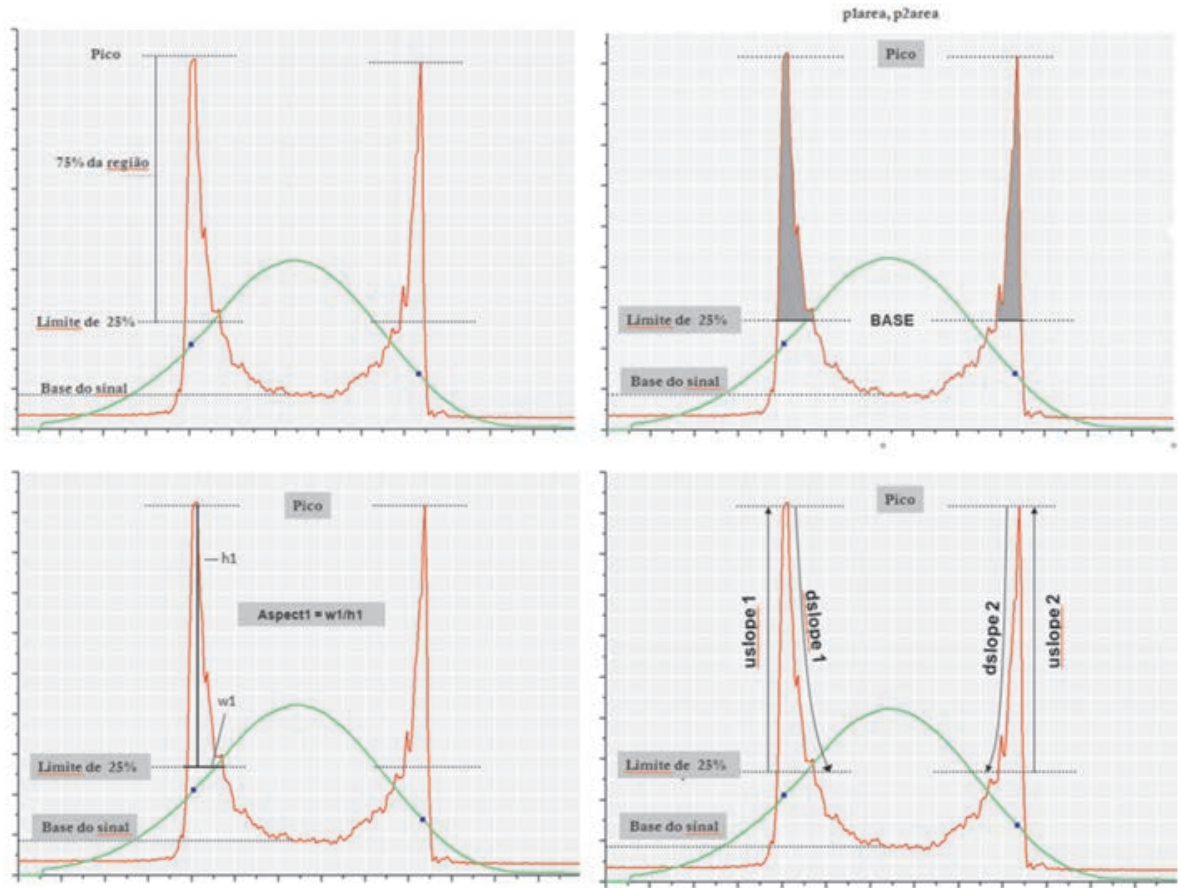

Nenhuma variável biomecânica apresentou diferença estatisticamente significante após a cirurgia (teste de Wilcoxon, $\mathrm{p}>0,05$ ).

Os parâmetros do ORA derivados das pressões de aplanamento: histerese da córnea (corneal hysteresis - $\mathrm{CH}$ ), fator de resistência da córnea (corneal resistance Factor-CRF) e as medidas da pressão ocular (IOPg e IOPcc) não apresentaram correlações significantes com os parâmetros delta $(\Delta)$ (teste de Wilcoxon, $p>0,05$ ).

Entretanto, 15 das 38 variáveis biomecânicas derivadas do sinal de reflexo da córnea do ORA apresentaram pelo menos uma correlação significante com um parâmetro $\Delta$, que reflete o resultado da cirurgia. O sumário de todas as correlações significantes encontra-se na tabela 4. Correlações com coeficiente positivo significam que o aumento de uma variável está relacionado com o aumento da outra. Portanto, neste estudo, no caso de correlações negativas, quanto menor o parâmetro préoperatório, maior foi o valor $\Delta$ em questão.

Destacam-se na figura 4 , as correlações entre o $\Delta$ KMáx com aindex e path1 (teste de Pearson, $\mathrm{p}<0,05$; $\mathrm{r}=-0,56$ e 0,56 respectivamente), entre o $\Delta \mathrm{AV} c \mathrm{cPré}-$ AVscPós e aindex (teste de Pearson, $\mathrm{p}<0,05 ; \mathrm{r}=0,48$ ).

A variável que teve maior número de correlações significantes foi $w 21$, que se correlacionou negativamente com $\Delta \mathrm{K} 2, \Delta \mathrm{K} 1, \Delta \mathrm{CKI}$ e $\Delta \mathrm{KMáx}$ (teste de

Figura 3 - Deslocamento vertical do KMáx no eixo Y

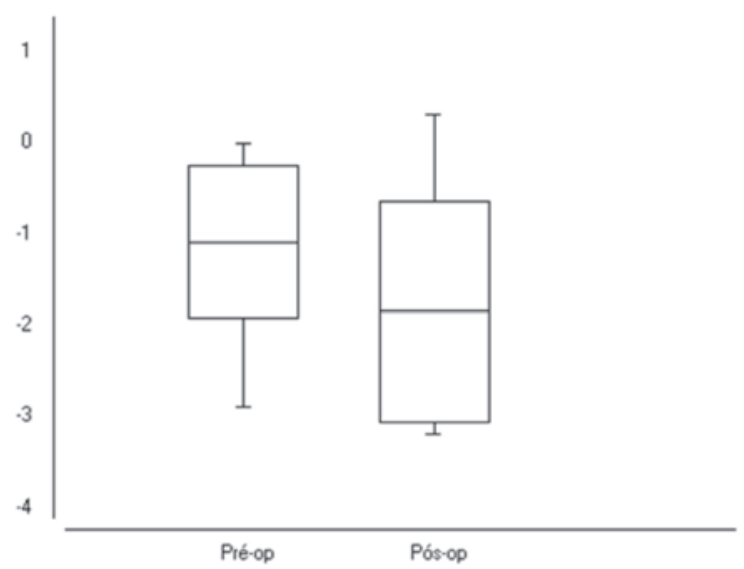

Spearman, $\mathrm{p}<0,05 ; \mathrm{rs}=-0,56 ;-0,55 ;-0,51$ e $-0,47$ respectivamente).

O deslocamento vertical do KMáx se relacionou positivamente com mslew 1 , aspect 21 , aspect 11 e slew 1 (teste de Pearson, $\mathrm{p}<0,05 ; \mathrm{r}=0,49 ; 0,47 ; 0,52 ; 0,48$ respectivamente) e negativamente com $w 1$ e $w 11$ (teste de Pearson, $\mathrm{p}<0,05 ; \mathrm{r}=-0,47$ e $-0,54$ respectivamente). 
Figura 4 - Correlações entre a mudança da Ceratometria mais elevada (KMáx), K2 e melhor acuidade visual corrigida (AVcc) e parâmetros biomecânicos
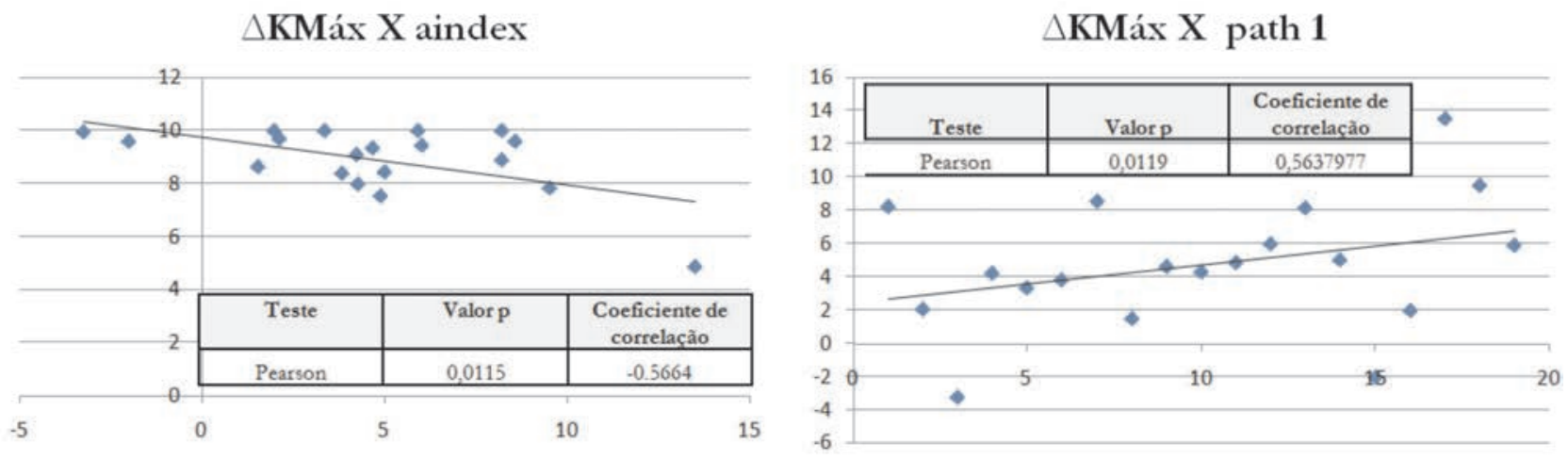

$\triangle \mathrm{AV} c$ Pré-AVscPós $\mathrm{x}$ aindex

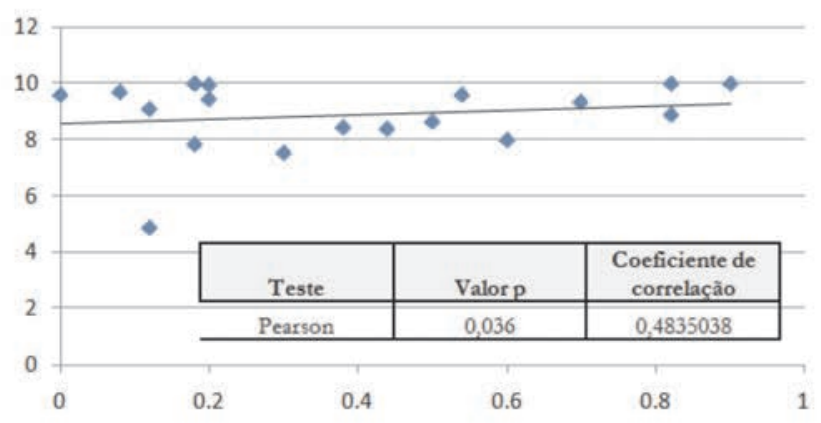

$\Delta \mathrm{K} 2 \mathrm{X}$ slew1

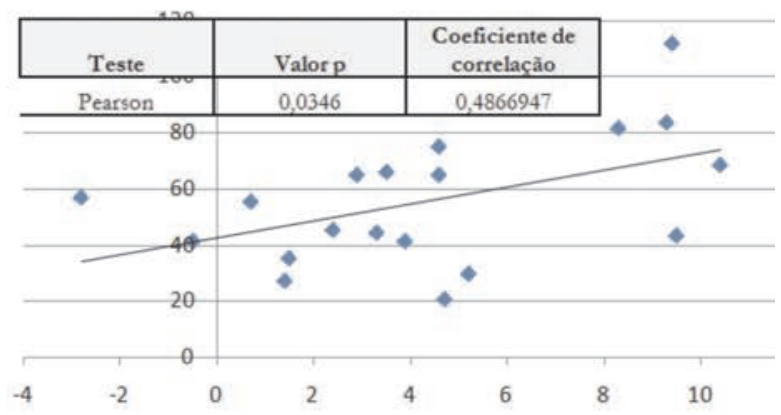

Figura 5 - Imagem de Scheimpflug, recomposição tomográfica e mapas paquimétricos pré, pós e diferencial

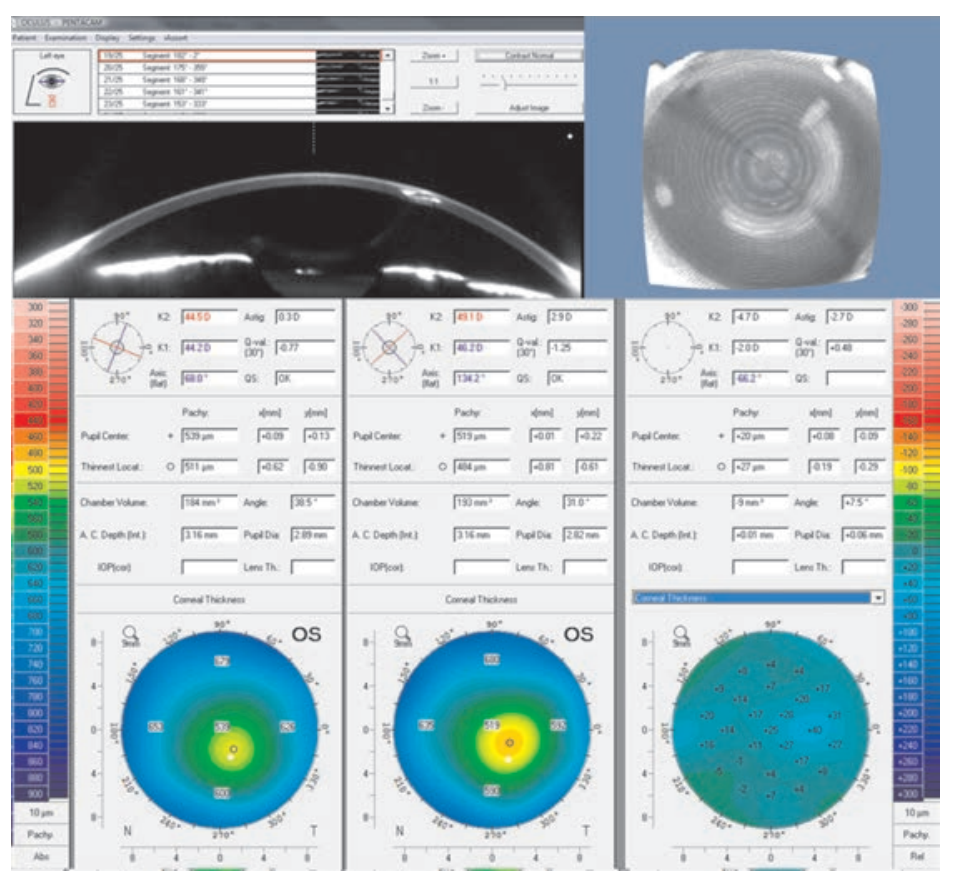


Figura 6 - Dois exemplos com evoluções distintas após cirurgias similares. Mapas de curvatura sagital pré, pós e diferencial e o sinal do ORA pré-operatório

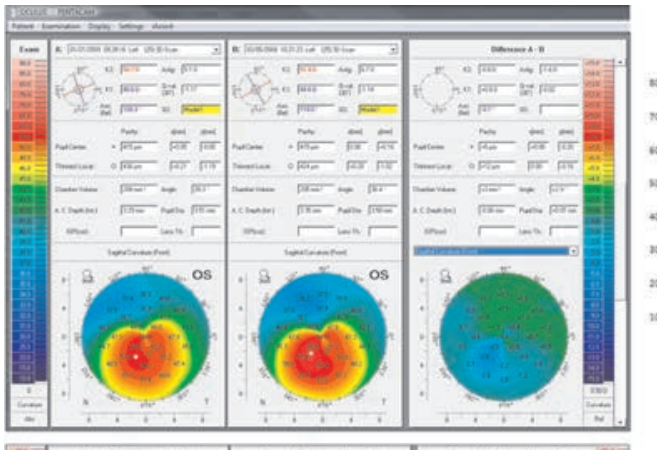

Exemplo 1
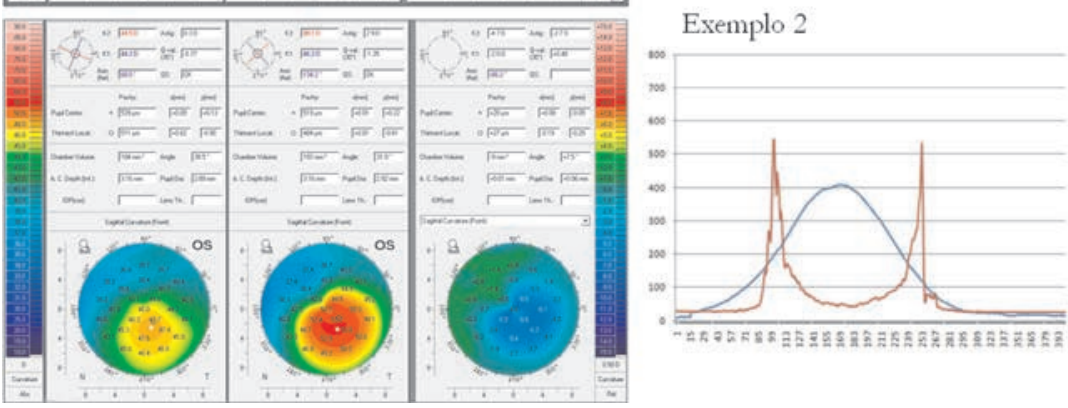

\section{DiscusSÃo}

Ceratocone é uma distrofia ectásica não inflamatória de caráter progressivo, caracterizada por afinamento e encurvamento da córnea, com protrusão cônica central ou paracentral, que ocorre por uma falência biomecânica do estroma da córnea que não é mais capaz de resistir às pressões internas e externas exercidas. $^{(31,32)}$

A doença determina redução da acuidade visual, bem como na qualidade de visão, o que afeta significativamente a qualidade de vida do paciente. ${ }^{(33-37)}$ No caso do tratamento da disfunção visual não ser adequado com óculos e inicialmente lentes de contato (LC) especiais, está indicada a cirurgia. As variações de ceratoplastia ou transplante de córnea eram as únicas cirurgias indicadas, sendo o ceratocone uma das causas mais frequentes de transplante de córnea. ${ }^{(38,39)}$ Entretanto, novas técnicas cirúrgicas como a promoção de ligações covalentes para reticulação do colágeno corneano (crosslinking), ${ }^{(40-43)} \mathrm{O}$ implante de segmentos de anéis intra-estromais, ${ }^{(5,11,44-47)}$ lentes fácicas, ${ }^{(48)}$ e técnicas de fotoablação ${ }^{(49-53)}$ são capazes de adiar ou mesmo evitar o transplante.

Por outro lado, existe uma tendência crescente de indicar o tratamento cirúrgico do ceratocone mais precocemente. Por exemplo, a indicação do crosslinking deve ser realizada para evitar a piora da visão, sendo fundamental uma propedêutica adequada para confirmar a indicação. Os avanços dos métodos diagnósticos em córnea, desde a topografia ${ }^{(23,54)}$ até a tomografia com reconstrução 3-D, ${ }^{(25,26,55,56)}$ o estudo biomecânico da córnea in vivo ${ }^{(29,57-61)}$ e a aberrometria ocular, ${ }^{(62-64)}$ se destacam também para o planejamento cirúrgico.

O implante de anel foi inicialmente desenvolvido para tratamento de miopia. ${ }^{(65)}$ Entretanto, o ceratocone se mostrou uma melhor indicação para este procedimento que adiciona material na periferia da córnea, com objetivo de induzir aplanamento central, o que também contribui para uma estabilidade estrutural.

Neste estudo, observamos a eficácia da cirurgia de implantes de segmentos de anel de Ferrara para melhorar a acuidade visual após a cirurgia. Além de haver diferença significante na acuidade visual corrigida, a visão sem correção após a cirurgia mostrou-se melhor que a corrigida antes da cirurgia. Entretanto, após a cirurgia, os pacientes apresentaram melhor AV com correção que sem correção, o que indica que mesmo após o tratamento cirúrgico, os pacientes podem se beneficiar de correção com óculos. A orientação adequada dos pacientes é fundamental para que entendam o papel da cirurgia, sem expectativas irreais de eliminação total dos erros refrativos. 
Observou-se melhora significativa nos valores ceratométricos e em diversos parâmetros topométricos após implante de segmentos de anel em casos de ceratocone. Adicionalmente, o estudo tomográfico foi eficiente para identificar aumento da espessura da córnea na região temporal, a 3,8 e $4 \mathrm{~mm}$ do ápice. Tal aumento de espessura reflete exatamente a posição do anel. Entretanto, não representa o aumento da espessura do anel, o que pode ser explicado por haver uma reorganização lamelar. Tal observação pode ser correlacionada com achados do mapa paquimétrico diferencial antes e após a cirurgia (Figura 5).

Nenhuma variável biomecânica apresentou diferença estatisticamente significante após a cirurgia (teste de Wilcoxon, $p>0,05)$. Tal observação corrobora com o conceito de que o implante de segmentos de anel não muda a estrutura da córnea, mas é capaz de equilibrar "ortopedicamente" a instabilidade ectásica, pois reduz o comprimento de arco das lamelas. A diminuição do arco reduz o "estresse" pressórico na córnea, equilibrando-a no seu aspecto biomecânico, o que contribui para estabilizar a progressão ectásica (afinamento e protrusão). Pode-se fazer uma analogia ao efeito de uma pestana no braço de um instrumento de cordas como o violão. O encurtamento da corda muda o som pela diferença na vibração acústica, mas não muda a composição da corda que mantém suas propriedades estruturais.

Os parâmetros $\Delta$ foram calculados para representar o resultado da cirurgia. Desse modo, foram possíveis estudos estatísticos das correlações entre o estado biomecânico pré-operatório e o efeito da cirurgia.

Os parâmetros derivados das pressões de aplanamento do ORA ( $\mathrm{CH}, \mathrm{CRF})$ e as medidas pressóricas (IOPg e IOPcc) não apresentaram correlações significantes com os parâmetros delta $(\Delta)$. Entretanto, 15 das 38 novas variáveis biomecânicas derivadas do sinal de reflexo da córnea do ORA apresentaram correlações significantes com pelo menos um parâmetro $\Delta$. Tal observação mostra que é fundamental atenção para as informações do sinal do ORA, que refletem o comportamento da córnea frente ao jato de ar. O estudo da deformação da córnea com o ORA se dá com o reflexo infravermelho que monitora a região de $1 \mathrm{~mm}$ em torno do ápice.

De forma geral, uma córnea com estrutura mais estável apresenta um sinal mais elevado e com menos irregularidade. A maioria dos parâmetros encontra-se reduzida em casos de ceratocone, com a exceção dos parâmetros path e aplhf, que representam a irregularidade do sinal do ORA durante a medida com o sopro de ar, e aumentam de acordo com a severidade da ectasia.

Observou-se deslocamento inferior significativo da posição do KMáx (teste de Wilcoxon, $\mathrm{p}<0,05$ ). A posição do KMáx no eixo vertical (Y) passou de $-1,09 \pm 0,84$ para $-1,85 \pm 1,22$, mas houve grande variação nesta mudança com a cirurgia, o que pode ser observado no gráfico de "box-plot" na Figura 3. De forma geral, o deslocamento inferior do ponto de maior curvatura reflete um benefício da cirurgia de implante de segmentos de anel, pois afasta a área de maior curvatura e maior irregularidade do centro da córnea e, por conseqüência, da área pupilar. A mudança de posição vertical do KMáx apresentou correlações significativas positivas com mslew1, aspect21, aspect11 e slew1. Correlações negativas foram encontradas com w1 e w11 (teste de Pearson, $\mathrm{p}<0,05$; $\mathrm{r}=-0,47$ e $-0,54$ respectivamente).

Enquanto a maioria das correlações foi negativa, corroborando com o fato que córneas mais fracas apresentam maior benefício com a cirurgia, houve correlações positivas com algumas variáveis, o que é paradoxal. Por exemplo, a variação do maior valor ceratométrico ( $\triangle$ KMáx) foi significante com aindex e path1, sendo positiva na primeira e negativa na segunda. A melhora da visão sem correção em relação a visão corrigida antes da cirurgia ( $\triangle \mathrm{AV} c c$ Pré-AVscPós) se correlacionou positivamente com o aindex (teste de Pearson, $\mathrm{p}<0,05$; $\mathrm{r}=0,48)$. A variável que teve maior número de correlações significantes foi w21, que se correlacionou negativamente com $\Delta \mathrm{K} 2, \Delta \mathrm{K} 1, \Delta \mathrm{CKI}$ e $\Delta \mathrm{KMáx}$ (teste de Spearman, $\mathrm{p}<0,05 ; \mathrm{r}=-0,56 ;-0,55 ;-0,51$ e $-0,47)$ respectivamente. Tal questão pode ser explicada pelo fato de que algumas características da deformação podem ser relacionadas com propriedades viscoelásticas e elásticas e quando estas estão menos baixas, uma maior previsibilidade do remodelamento com o implante de anel é observada.

Existem, portanto, diversas correlações de características biomecânicos da córnea com ceratocone no pré-operatório com o resultado da cirurgia. Nenhum estudo publicado ou apresentado em congressos de Oftalmologia reporta achados similares, sendo este o primeiro relato da literatura mundial que refere a influencia da biomecânica da córnea no resultado da cirurgia de implante de segmentos de anel estromal.

A relevância destes achados pode ser demonstrada com os exemplos de dois pacientes com evolução bastante diferente após cirurgias similares e aspectos topográficos pré-operatórios similares (Figura 6). Enquanto o exemplo 1 apresentou um efeito mais irregular com pequena redução do astigmatismo e astigmatismo 
residual superior a 5DC, o exemplo 2 apresentou uma maior correção do astigmatismo e um astigmatismo residual de 0,3DC. Observam-se diferenças no sinal do ORA pré-operatório em ambos os casos.

As correlações foram significativas com coeficientes em torno de 0.5 (média dos valores absolutos foi 0,50 , variando entre 0,46 e 0,57). Estudos com análises multivariadas são necessários para criar um modelo que traga uma possível equação com base em parâmetros biomecânicos para determinar uma correlação maior. Tal estudo fornecerá maior precisão para fatores prognósticos do implante de segmentos de anel para ceratocone. Tais observações deverão ser utilizadas para aprimorar os nomogramas destas cirurgias. O presente trabalho demonstra estatisticamente diversas correlações entre o aspecto biomecânico pré-operatório e o resultado da cirurgia de implante de segmentos de anel estromal, o que abre novos horizontes para o uso destas variáveis com o objetivo de melhorar o planejamento das cirurgias para ceratocone.

\section{REFERÊNCIAS}

1. Alió JL, Piñero DP, Sögütlü E, Kubaloglu A. Implantation of new intracorneal ring segments after segment explantation for unsuccessful outcomes in eyes with keratoconus. J Cataract Refract Surg. 2010;36(8):1303-10.

2. AlióJL, Shabayek MH, Artola A. Intracorneal ring segments for keratoconus correction: long-term follow-up. J Cataract Refract Surg. 2006;32(6):978-85.

3. Alió JL, Shabayek MH, Belda JI, Correas P, Feijoo ED. Analysis of results related to good and bad outcomes of Intacs implantation for keratoconus correction. J Cataract Refract Surg. 2006;32(5):756-61.

4. Boxer Wachler BS, Christie JP, Chandra NS, Chou B, Korn T, Nepomuceno R. Intacs for keratoconus. Ophthalmology. 2003;110(5):1031-40. Erratum in Ophthalmology. 2003;110(8):1475.

5. Colin J, Cochener B, Savary G, Malet F. Correcting keratoconus with intracorneal rings. J Cataract Refract Surg. 2000;26(8):1117-22.

6. Coskunseven E, Kymionis GD, Grentzelos MA, Karavitaki AE, Portaliou DM, Jankov MR 2nd, Arslan E. INTACS followed by KeraRing intrastromal corneal ring segment implantation for keratoconus. J Refract Surg. 2010;26(5):371-4.

7. Daxer A, Mahmoud H, Venkateswaran RS. Intracorneal continuous ring implantation for keratoconus: One-year followup. J Cataract Refract Surg. 2010;36(8):1296-302.

8. Ertan A, Colin J. Intracorneal rings for keratoconus and keratectasia. J Cataract Refract Surg. 2007;33(7):1303-14.

9. Ertan A, Kamburoglu G, Bahadir M. Intacs insertion with the femtosecond laser for the management of keratoconus: oneyear results. J Cataract Refract Surg. 2006;32(12):2039-42.

10. Hellstedt T, Mäkelä J, Uusitalo R, Emre S, Uusitalo R. Treating keratoconus with intacs corneal ring segments. J Refract Surg. 2005;21(3):236-46.

11. Kwitko S, Severo NS. Ferrara intracorneal ring segments for keratoconus. J Cataract Refract Surg. 2004;30(4):812-20.
12. Kymionis GD, Siganos CS, Tsiklis NS, Anastasakis A, Yoo SH, Pallikaris AI, et al. Long-term follow-up of Intacs in keratoconus. Am J Ophthalmol. 2007;143(2):236-44.

13. Piñero DP, Alio JL. Intracorneal ring segments in ectatic corneal disease - a review. Clin Experiment Ophthalmol. 2010;38(2):154-67. Review.

14. Jhanji V, Sharma N, Vajpayee RB. Management of keratoconus: current scenario. Br J Ophthalmol. 2010 Aug 7. [Epub ahead of print].

15. El Dib RP, de Freitas D. A systematic review of Ferrara's ring in the treatment of keratoconus. J Refract Surg. 2008;24(9):865-6.

16. Tan DT, Por YM. Current treatment options for corneal ectasia. Curr Opin Ophthalmol. 2007;18(4):284-9.

17. Güell JL. Are intracorneal rings still useful in refractive surgery? Curr Opin Ophthalmol. 2005;16(4):260-5.

18. Colin J, Velou S. Current surgical options for keratoconus. J Cataract Refract Surg. 2003;29(2):379-86.

19. Pesando PM, Ghiringhello MP, Di Meglio G, Romeo S. Treatment of keratoconus with Ferrara ICRS and consideration of the efficacy of the Ferrara nomogram in a 5-year follow-up. Eur J Ophthalmol. 2010;20(5):865-73.

20. Moreira H, Oliveira CS, Godoy G, Wahab SA. Anel intracorneano de Ferrara em ceratocone. Arq Bras Oftalmol. 2002;65(1):59-63.

21. Piñero DP, Alio JL, Teus MA, Barraquer RI, Uceda-Montañés A. Modeling the intracorneal ring segment effect in keratoconus using refractive, keratometric, and corneal aberrometric data. Invest Ophthalmol Vis Sci. 2010;51(11):5583-91.

22. Piñero DP, Alió JL, Teus MA, Barraquer RI, Michael R, Jiménez $\mathrm{R}$. Modification and refinement of astigmatism in keratoconic eyes with intrastromal corneal ring segments. J Cataract Refract Surg. 2010;36(9):1562-72.

23. Klyce SD. Computer-assisted corneal topography. High-resolution graphic presentation and analysis of keratoscopy. Invest Ophthalmol Vis Sci. 1984;25(12):1426-35.

24. Wilson SE, Ambrosio R. Computerized corneal topography and its importance to wavefront technology. Cornea. 2001;20(5):441-54.

25. Ambrósio R Jr, Belin MW. Imaging of the cornea: topography vs tomography. J Refract Surg. 2010;26(11):847-9.

26. Ambrósio R Jr, Alonso RS, Luz A, Coca Velarde LG. Cornealthickness spatial profile and corneal-volume distribution: tomographic indices to detect keratoconus. J Cataract Refract Surg. 2006;32(11):1851-9.

27. Fontes BM, Ambrósio R Jr, Jardim D, Velarde GC, Nosé W. Corneal biomechanical metrics and anterior segment parameters in mild keratoconus. Ophthalmology. 2010;117(4):673-9.

28. Fontes BM, Ambrósio R Jr, Salomão M, Velarde GC, Nosé W. Biomechanical and tomographic analysis of unilateral keratoconus. J Refract Surg. 2010;26(9):677-81.

29. Luce DA. Determining in vivo biomechanical properties of the cornea with an ocular response analyzer. J Cataract Refract Surg. 2005;31(1):156-62.

30. Charman WN. Wavefront technology: past, present and future. Cont Lens Anterior Eye. 2005;28(2):75-92.

31. Rabinowitz YS. Keratoconus. Surv Ophthalmol. 1998;42(4):297-319. Review.

32. Krachmer JH, Feder RS, Belin MW. Keratoconus and related noninflammatory corneal thinning disorders. Surv Ophthalmol. 1984;28(4):293-322. Review.

33. Szczotka LB, Barr JT, Zadnik K. A summary of the findings from the Collaborative Longitudinal Evaluation of Keratoconus (CLEK) Study. CLEK Study Group. Optometry. 2001;72(9):574-84.

34. Moreira LB, Alchieri JC, Belfort Júnior R, Moreira H. Aspectos psicossociais do paciente com ceratocone. Arq Bras Oftalmol. 2007;70(2):317-22. 
35. Kymes SM, Walline JJ, Zadnik K, Sterling J, Gordon MO; Collaborative Longitudinal Evaluation of Keratoconus Study Group. Changes in the quality-of-life of people with keratoconus. Am J Ophthalmol. 2008;145(4):611-7.

36. Kymes SM, Walline JJ, Zadnik K, Gordon MO; Collaborative Longitudinal Evaluation of Keratoconus study group. Quality of life in keratoconus. Am J Ophthalmol. 2004;138(4):527-35.

37. de Freitas Paranhos J, Avila MP, Paranhos A Jr, Schor P. Evaluation of the impact of intracorneal ring segments implantation on the quality of life of patients with keratoconus using the NEI-RQL (National Eye Institute Refractive Error Quality of life) instrument. Br J Ophthalmol. 2010;94(1):101-5.

38. Flowers CW, Chanq KY, McLeod SD, Irvine JA, McDonnell PJ, Rao N, Smith RE. Changing indications for penetrating keratoplasty, 1989-1993. Cornea. 1995;14(6):583-8.

39. Ghosheh FR, Cremona FA, Rapuano CJ, Cohen EJ, Ayres $\mathrm{BD}$, Hammersmith KM, et al. Trends in penetrating keratoplasty in the United States 1980-2005. Int Ophthalmol. 2008;28(3):147-53.

40. Jankov II MR, Hafezi F, Beko M, Ignjatovic Z, Djurovic B, Markovic V, Schor P. Ultra B2 - Promoção de ligações covalentes do colágeno corneal (Corneal cross-linking) no tratamento de ceratocone: resultados preliminares. Arq Bras Oftalmol. 2008;71(6):813-8.

41. Lamy R, Netto CF, Pecego MG, Pecego JGC, Pereira BB, Moraes Júnior HV, Dantas AM. Reticulação do colágeno corneano com radiação ultravioleta e riboflavina para tratamento do ceratocone: resultados preliminares de um estudo brasileiro. Rev Bras Oftalmol. 2008;67(5):231-5

42. Wollensak G, Spoerl E, Seiler T. Riboflavin/ultraviolet-a-induced collagen crosslinking for the treatment of keratoconus. Am J Ophthalmol. 2003;135(5):620-7.

43. Iovieno A, Oechsler RA, Yoo SH. Long-term results of collagen crosslinking with riboflavin and UVA in keratoconus. J Cataract Refract Surg. 2008;34(10):1616-7; author reply 1617.

44. Piñero DP, Alio JL, El Kady B, Coskunseven E, Morbelli H, Uceda-Montanes A, et al. Refractive and aberrometric outcomes of intracorneal ring segments for keratoconus: mechanical versus femtosecond-assisted procedures. Ophthalmology. 2009;116(9):1675-87.

45. Alió JL, Shabayek MH. Intracorneal asymmetrical rings for keratoconus: where should the thicker segment be implanted? J Refract Surg. 2006;22(3):307-9.

46. Colin J, Simonpoli-Velou S. The management of keratoconus with intrastomal corneal rings. Int Ophthalmol Clin. 2003;43(3):65-80.

47. Siganos D, Ferrara P, Chatzinikolas K, Bessis N, Papastergiou G. Ferrara intrastromal corneal rings for the correction of keratoconus. J Cataract Refract Surg. 2002;28(11):1947-51.

48. Leccisotti A, Fields SV. Angle-supported phakic intraocular lenses in eyes with keratoconus and myopia. J Cataract Refract Surg. 2003;29(8):1530-6.

49. Kymionis GD, Kontadakis GA, Kounis GA, Portaliou DM, Karavitaki AE, Magarakis M, et al. Simultaneous topography-guided PRK followed by corneal collagen cross-linking for keratoconus. J Refract Surg. 2009;25(9):S807-11.

50. Kanellopoulos AJ. Comparison of sequential vs same-day simultaneous collagen cross-linking and topography-guided PRK for treatment of keratoconus. J Refract Surg. 2009;25(9):S812-8.
51. Lin DT, Holland SR, Rocha KM, Krueger RR. Method for optimizing topography-guided ablation of highly aberrated eyes with the ALLEGRETTO WAVE excimer laser. J Refract Surg. 2008;24(4):S439-45.

52. Cennamo G, Intravaja A, Boccuzzi D, Marotta G, Cennamo G. Treatment of keratoconus by topography-guided customized photorefractive keratectomy: two-year follow-up study. J Refract Surg. 2008;24(2):145-9.

53. Kanellopoulos AJ, Binder PS. Collagen cross-linking (CCL) with sequential topography-guided PRK: a temporizing alternative for keratoconus to penetrating keratoplasty. Cornea. 2007;26(7):891-5.

54. Ambrósio R Jr, Klyce SD, Wilson SE. Corneal topographic and pachymetric screening of keratorefractive patients. J Refract Surg. 2003;19(1):24-9.

55. Wolf A, Abdallat W, Kollias A, Frohlich SJ, Grueterich M, Lackerbauer CA. Mild topographic abnormalities that become more suspicious on Scheimpflug imaging. Eur J Ophthalmol. 2009;19(1):10-7.

56. de Sanctis U, Loiacono C, Richiardi L, Turco D, Mutani B, Grignolo FM. Sensitivity and specificity of posterior corneal elevation measured by Pentacam in discriminating keratoconus/subclinical keratoconus. Ophthalmology. 2008;115(9):1534-9.

57. Fontes BM, Ambrósio R Jr, Alonso RS, Jardim D, Velarde GC, Nosé W. Corneal biomechanical metrics in eyes with refraction of -19.00 to $+9.00 \mathrm{D}$ in healthy Brazilian patients. J Refract Surg. 2008;24(9):941-5.

58. Fontes BM, Ambrósio R Jr, Velarde GC, Nosé W. Corneal biomechanical evaluation in healthy thin corneas compared with matched keratoconus cases. Arq Bras Oftalmol. 2011;74(1):13-6.

59. Dupps WJ Jr. Hysteresis: new mechanospeak for the ophthalmologist. J Cataract Refract Surg. 2007;33(9):1499-501. Comment on J Cataract Refract Surg. 2007;33(9):1530-8.

60. Kotecha A. What biomechanical properties of the cornea are relevant for the clinician? Surv Ophthalmol. 2007;52 Suppl 2:S109-14.

61. Shah S, Laiquzzaman M, Bhojwani R, Mantry S, Cunliffe I. Assessment of the biomechanical properties of the cornea with the ocular response analyzer in normal and keratoconic eyes. Invest Ophthalmol Vis Sci. 2007;48(7):3026-31.

62. Okamoto C, Okamoto F, Samejima T, Miyata K, Oshika T. Higher-order wavefront aberration and letter-contrast sensitivity in keratoconus. Eye (Lond). 2008;22(12):1488-92.

63. Jafri B, Li X, Yang H, Rabinowitz YS. Higher order wavefront aberrations and topography in early and suspected keratoconus. J Refract Surg. 2007;23(8):774-81.

64. Marcos S. Aberrometry: basic science and clinical applications. Bull Soc Belge Ophtalmol. 2006;(302):197-213.

65. Cunha PFA. Técnica cirúrgica para correção de miopia. Anel corneano intra-estromal. Rev Bras Oftalmol. 1995;54(8):577-88.
Endereço para Correspondência:
Dr. Renato Ambrósio Jr.
Rua Conde de Bonfim, ${ }^{\circ} 211$ apto 712 - Tijuca
CEP 20.520-050 - - Rio de Janeiro (RJ), Brasil
Telefone: (21) 2234 4233/2274 5694/ 78922131
E-mail: renatoambrosiojr@terra.com.br 